\title{
Work Resumption After Epidemic Using Three-Way Decisions
}

\author{
Xiang $\mathrm{Li}^{1} \cdot$ Hai Wang ${ }^{2} \cdot$ Zeshui $\mathrm{Xu}^{1,3}$
}

Received: 4 July 2020/Revised: 29 September 2020/Accepted: 9 November 2020/Published online: 5 January 2021

(C) Taiwan Fuzzy Systems Association 2021

\begin{abstract}
After the epidemic, all enterprises are faced with the difficult decision about whether the enterprise should resume work and production immediately, which is related to the safety and development of enterprises. The threeway decisions offer a novel study perspective to solve this problem. Firstly, we describe some relevant attributes of the enterprise with double hierarchy hesitant fuzzy linguistic term sets, and construct double hierarchy hesitant fuzzy linguistic information systems for each enterprise. Secondly, we get the weights of attributes with the entropy weight method, and take the weighted aggregation of attributes information as the conditional probability that the enterprise is in a safe state. Next, we classify each enterprise according to its size. Considering the influence of different sizes of enterprises, we put forward the corresponding loss function matrix. Then we get the decision results about work resumption based on the principle of minimizing the loss, which demonstrates the practicability and effectiveness of our method. Finally, we compare the method proposed by us with the other method and discuss the advantages and limitations of our proposed method.
\end{abstract}

Zeshui Xu

xuzeshui@263.net

Xiang Li

1x19951818@163.com

Hai Wang

wanghai@nau.edu.cn

1 School of Economics and Management, Southeast University, Nanjing 210007, Jiangsu, China

2 School of Information Engineering, Nanjing Audit University, Nanjing 211815, Jiangsu, China

3 Business School, Sichuan University, Chengdu 610064, China
Keywords Work resumption - Three-way decisions (TWDs) · Double hierarchy hesitant fuzzy linguistic term sets (DHHFLTSs) · Entropy weight method

\section{Introduction}

Since the novel coronavirus broke out on the worldwide scale, the world economy has been seriously hurt [1-3]. At the beginning of 2020, almost all enterprises and individual businesses in China halted production and business for more than a month, which caused about 2.5 trillion yuan of direct losses to economic activities. The manufacturing industry fell by 13 percent between January and February according to the Bureau of Statistics. After the novel coronavirus was controlled, the resumption of work and production [4] has been put on the agenda, which is of great significance to the recovery of economic development. On February 9, 2020, 30 other provinces except Hubei Province in China have arranged for enterprises to resume work and production. But when we resume work and production, we also need to ensure the health and safety of our employees. Therefore, all enterprises are confronted with the difficulties of making the decision that whether to resume work and production immediately.

The three-way decisions (TWDs) offer a novel study perspective to solve this problem. It attracted the attention of scholars since their proposal by Yao [5, 6] in 2010. Intuitively, it divides objects into three disjoint regions (positive region, boundary region, and negative region) based on the Bayesian process. When an object is divided into these three fields, it represents accepting the object, hesitating, and rejecting the object, respectively. As the three-way decisions fit the human's thinking patterns, it can be applied to many fields like cluster analysis [7, 8], risk 
decision-making [9, 10], credit scoring [11], etc. In order to describe the loss function in the three-way decisions better, many scholars have introduced fuzzy set theory [12] into the process of TWDs. Liang and Liu [13, 14] used triangle fuzzy numbers and intuitionistic fuzzy sets to describe the loss function, respectively, and created the triangular fuzzy decision-theoretic rough sets (TFDTRSs) model and intuitionistic fuzzy decision-theoretic rough sets (IFDTRSs) model. Liang and $\mathrm{Xu}$ et al. [15] described the loss function with dual hesitant fuzzy information and studied some properties of the model. Liang and Pedrycz et al. [16] studied three-way group decisions method under linguistic terms set environment. Yang and Yao [17] deal with uncertainty and incomplete information by TWDs with soft sets. Ye and Zhan et al. [18] proposed a novel TWDs method by constructing fuzzy $\varepsilon$-neighborhood classes. Liang and Xu et al. [19] developed the method for TWDs at Pythagorean fuzzy information and proposed using ideal TOPSIS solutions for the calculation of conditional probability.

Because the linguistic terms conform with people's habits of expression more, Zadeh [20] puts forward the definition of Computing with Words (CW) and account for its meaning. After that, some linguistic models based on CW like the hesitant fuzzy linguistic term set (HFLTS) [21], the 2-tuple linguistic model [22, 23], and the virtual linguistic model [24] have been developed. To improve the accuracy of linguistic terms, Gou et al. [25, 26] proposed the definition of double hierarchy fuzzy linguistic term set (DHFTS). Combined with the merits of hesitant fuzzy sets, Gou et al. [25] developed DHHFLTSs, The appearance of DHHFLTSs provides a novel tool for describing loss functions in TWDs.

Based on the principle of preventing viruses scientifically and implementing the method precisely, all enterprises are faced with the difficult decision about whether the enterprise should resume work and production immediately, which is related to the safety and development of enterprises. The three-way decisions meet the decisionmaking needs of enterprises. It offers the enterprises three options: return to work immediately, delay decisions, and suspend resuming work temporarily. Combined with the actual situation of the enterprise, the risk probability of infection will be calculated after the resumption of work. And the decision results will minimize the losses caused by the epidemic to enterprises. The DHHFLTS consists of two hierarchies completely independent of LTSs. As a kind of linguistic term, DHHFLTS conforms to people's expression habits. In contrast to the single linguistic term set, DHHFLTSs provide more flexible manner to express qualitative information by means of linguistic expressions. When experts evaluate the information of attributes and the loss degree of the enterprise, they can use it intuitively to give the evaluation value, which saves a lot of time for decision-making. It is a kind of convenient and effective tool for experts, which let the experts make decisions more easily.

On the basis of the above analysis, the merits of the proposed method are as follows: (1) applies the TWDs method to the decision-making of work resumption after the epidemic; (2) tries to use DHHFLTSs to describe the loss functions in TWDs; (3) obtains the weight of each attribute by the entropy weight method, and take the weighted aggregation of attribute information as the conditional probability; (4) puts forward the corresponding loss function matrixes in light of the different sizes of companies.

The main content of this paper is organized as follows: In Sect. 2, we provide the basic concepts of DHHFLTSs. In Sect. 3, we discuss a novel DTRSs model with DHHFLT expression of loss functions. In Sect. 4, we introduce the calculation process of conditional probability in the decision-making problem of work resumption. In Sect. 5, we design decision-making process of the method proposed by us. In Sect. 6, a case study of work resumption after the epidemic is given. In Sect. 7, we compare the method proposed by us with the other method and discuss the advantages and limitations of the method proposed by us. In Sect. 8, we conclude the study and further elaborates its theoretical and applied value.

\section{Double Hierarchy Hesitant Fuzzy Linguistic Term Sets}

According to the result reported in Ref. [25], each DHHFLTS consists of two hierarchies completely independent of LTSs. Next, we recall some basic concepts of DHHFLTSs.

Sometimes, people need to describe the sentences more accurately and in detail. To meet people's demand, Gou et al. [25] proposed the definition of the DHLTS.

Definition 2.1 [25] Given that $S=\left\{s_{t} \mid t=-\tau, \ldots,-1,0\right.$, $1, \ldots, \tau\}$ and $O=\left\{o_{k} \mid k=-\zeta, \ldots,-1,0,1, \ldots, \zeta\right\}$ are the first and second hierarchies of LTS, respectively, and they do not affect each other completely. Then, the mathematical form of DHLTS, $S_{o}$, is shown as follows:

$$
\begin{gathered}
S_{o}=\left\{s_{t\left\langle o_{k}\right\rangle} \mid t=-\tau, \ldots,-1,0,1, \ldots,\right. \\
\tau ; k=-\zeta, \ldots,-1,0,1, \ldots, \zeta\}
\end{gathered}
$$

among them, $s_{t\left\langle o_{k}\right\rangle}$ denotes the DHLT, $s_{t}$ represents the first hierarchy LT, $o_{k}$ represents the second hierarchy LT.

It is worth noting that the order of the second hierarchy LTS needs to be displayed in the light of the values of $t$. 
Remark 2.1 [25] There are four types of propositions shown based on different values of $\mathrm{t}$ : (1) If $t>0$, that is the first hierarchy LTS is positive, the second hierarchy LTS needs to be displayed in ascending order. (2) If $t<0$, that is the first hierarchy LTS is negative, the second hierarchy LTS needs to be displayed in descending order inversely. (3) If $t=\tau$, then we just take into account the front half of the second hierarchy LTS. (4) If $t=-\tau$, then we take into account the latter half of the second hierarchy LTS.

To make it easier to deal with DHLT, Gou et al. [25] proposed the definition of two transformed functions between the numerical scale and the subscript of the DHLT.

Definition 2.2 [25] Given that $\bar{S}_{o}=\left\{s_{t\left\langle o_{k}\right\rangle} \mid t=[-\tau, \tau] ; k=\right.$ $[-\zeta, \zeta]\}$ is a virtual DHLTS, $h_{S_{o}}=\left\{s_{\phi_{l}<o_{\varphi_{l}}}>\mid s_{\phi_{l}<o_{\varphi_{l}}}>\in\right.$ $\left.S_{o} ; l=1,2, \ldots, L ; \phi_{l}=[-\tau, \tau] ; \varphi_{l}=[-\zeta, \zeta]\right\}$ is a double hierarchy hesitant fuzzy linguistic element (DHHFLE), and $h_{\gamma}=\left\{\gamma_{l} \mid \gamma_{l} \in[0,1] ; l=1,2, \ldots, L\right\}$ is a set of numerical scales. There are two transformed functions $f$ and $f^{-1}$ between the numerical scale and the subscript $\left(\phi_{l}, \varphi_{l}\right)$ of the DHLT $s_{\phi_{l}<o_{\varphi_{l}}}>$ :

$$
\begin{aligned}
& f:[-\tau, \tau] \times[-\varsigma, \varsigma] \rightarrow[0,1], \\
& f\left(\phi_{l}, \varphi_{l}\right)=\frac{\varphi_{l}+\left(\tau+\phi_{l}\right) \varsigma}{2 \varsigma \tau}=\gamma_{l} \\
& f^{-1}:[0,1] \rightarrow[-\tau, \tau] \times[-\varsigma, \varsigma], \\
& f^{-1}\left(\gamma_{l}\right)=\left[2 \tau \gamma_{l}-\tau\right]<o_{\varsigma\left(2 \tau \gamma_{l}-\tau-\left[2 \tau \gamma_{l}-\tau\right]\right)}> \\
& \quad=\left[2 \tau \gamma_{l}-\tau\right]+1<o_{\zeta\left(\left(2 \tau \gamma_{l}-\tau-\left[2 \tau \gamma_{l}-\tau\right]\right)-1\right)}>
\end{aligned}
$$

On the basis of Definition 2.2, we can develop the transformation functions $\mathrm{F}$ and $F^{-1}$ between the DHFT $S_{\phi_{l}<o_{\varphi_{l}}}>$ and the numerical scale $\gamma_{l}$.

$$
\begin{aligned}
& F: \bar{S}_{o} \rightarrow h_{\gamma}, F\left(s_{\phi_{l}<o_{\varphi_{l}}}>\right)=f\left(\phi_{l}, \varphi_{l}\right)=\gamma_{l} \\
& F^{-1}: h_{\gamma} \rightarrow \bar{S}_{o}, F^{-1}\left(\gamma_{l}\right)=f^{-1}\left(\gamma_{l}\right)=s_{\phi_{l}<o_{\varphi_{l}}}>
\end{aligned}
$$

Combined with hesitant fuzzy environment, Gou et al. [25] proposed the definition of DHHFLTSs.

Definition 2.3 Gou et al. [25] Given that $X$ is a fixed set, $S_{o}=\left\{s_{t\left\langle o_{k}\right\rangle} \mid t=-\tau, \ldots,-1,0,1, \ldots, \tau ; k=-\zeta, \ldots,-1,0,1, \ldots, \zeta\right\}$

is a DHLTS. $H_{S_{o}}$ denotes a DHHFLTSs on $X$. It is expressed by a membership function. The function applied to $\mathrm{X}$ returns a subset of $S_{o}$, and its mathematical form is shown as follows:

$$
H_{S_{o}}=\left\{<x_{i}, h_{S_{o}\left(x_{i}\right)}>\mid x_{i} \in X\right\}
$$

among them, $h_{S_{o}\left(x_{i}\right)}$ is a set of some values in $S_{o}$, denoted as follows:

$$
\begin{aligned}
& h_{S_{o}}\left(x_{i}\right)=\left\{s_{\phi_{l}<o_{\varphi_{l}}}>\left(x_{i}\right) \mid s_{\phi_{l}<o_{\varphi_{l}}}>\in S_{o} ; l\right. \\
& \quad=1,2, \ldots, L ; \phi_{l}=-\tau, \ldots,-1,0,1, \ldots, \tau ; \\
& \left.\quad \varphi_{l}=-\zeta, \ldots,-1,0,1, \ldots, \zeta\right\}
\end{aligned}
$$

where $\mathrm{L}$ denotes the number of DHLTs in $h_{S_{o}}\left(x_{i}\right)$ and $s_{\phi_{l}<o_{\varphi_{l}}}>\left(x_{i}\right)(l=1,2, \ldots, L)$ in each $h_{S_{o}}\left(x_{i}\right)$ are the continuous terms in $S_{o} . h_{S_{o}}\left(x_{i}\right)$ represents the possible degree of the linguistic variable $x_{i}$ to $S_{o}$. Then, we call $h_{S_{o}}\left(x_{i}\right)$ the DHHFLE, and $\phi \times \psi$ is the set of all DHHFLEs.

Because the numbers of DHLTs in DHHFLEs may be different, Gou et al. [27] developed the concept of linguistic expected value for implementing the normalization process.

Definition 2.4 [27] Given that $\bar{S}_{o}=\left\{s_{t\left\langle o_{k}\right\rangle} \mid t=\right.$ $[-\tau, \ldots, \tau] ; k=[-\zeta, \ldots, \zeta]\}$ is a continuous DHLTS, $h_{S_{o}}=\left\{s_{\phi_{l}<o_{\varphi_{l}}}>\mid s_{\phi_{l}<o_{\varphi_{l}}}>\in \bar{S}_{o} ; l=1,2, \ldots, L\right\} \quad$ is $\quad$ a DHHFLE, $\phi \times \psi$ is the set of all DHHFLEs over $\bar{S}_{o}$. Then, we can attain a linguistic expected value of $h_{S_{o}}$ as follows:

$$
\begin{aligned}
& l e: \phi \times \psi \rightarrow \bar{S}_{o}, l e\left(h_{s_{o}}\right)=\frac{1}{L} \bigoplus_{l=1}^{L} s_{\phi_{l}<o_{\varphi_{l}}}>
\end{aligned}
$$

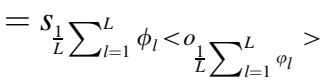

Next, we will describe the loss functions in the TWDs by DHHFLTSs.

\section{A New DTRSs Model with DHHFLT Expression of Loss Functions}

As the introduction of DHHFLTSs [25], it consists of two hierarchies completely independent of LTSs and improves the accuracy of linguistic expression. In this section, we try to use DHHFLTSs to describe the loss functions in TWDs and propose a novel DTRSs model with DHHFLT information. There are two states and three actions in the new DTRS model. $\Omega=\left\{X, X^{c}\right\}$ represents the set of states which manifests that an element is in $X$ or not in $X$. As for three actions, $\Lambda=\left\{a_{P}, a_{B}, a_{N}\right\}$ denotes the set of actions, and they are used to classify an object $x$. $a_{P}$ represents $x_{i} \epsilon \operatorname{POS}(X), a_{B}$ represents $x_{i} \in B N D(X)$, and $a_{N}$ represents $x_{i} \in N E G(X)$, where $P O S(X), B N D(X), N E G(X)$ denote the positive region, the boundary region, and the negative region of $X$, respectively. The states represent overall situation of the object and the actions represent our judgements. We construct the loss function matrix under the DHHFLT environment. The results are displayed as in Table 1. 
Table 1 The DHHFLT loss function about the actions in different states

\begin{tabular}{lll}
\hline & $\mathrm{X}(\mathrm{P})$ & $X^{c}(N)$ \\
\hline$a_{P}$ & $h_{S_{o}}^{\lambda_{P P}}=\left\{S_{\phi_{l}<o_{\varphi_{l}}}^{\lambda_{P P}}\right\}$ & $h_{S_{o}}^{\lambda_{P N}}=\left\{S_{\phi_{l}<o_{\varphi_{l}}}^{\lambda_{P_{N}}}\right\}$ \\
$a_{B}$ & $h_{S_{o}}^{\lambda_{B P}}=\left\{S_{\phi_{l}<o_{\varphi_{l}}}^{\lambda_{B P}}\right\}$ & $h_{S_{o}}^{\lambda_{B N}}=\left\{S_{\phi_{l}<o_{\varphi_{l}}}^{\lambda_{B N}}\right\}$ \\
$a_{N}$ & $h_{S_{o}}^{\lambda_{N P}}=\left\{S_{\phi_{l}<o_{\varphi_{l}}}^{\lambda_{N P}}\right\}$ & $h_{S_{o}}^{\lambda_{N_{N}}}=\left\{S_{\phi_{l}<o_{\varphi_{l}}}^{\lambda_{N N}}\right\}$ \\
\hline
\end{tabular}

From Table 1, we can attain that the loss functions $h_{S_{o}}^{\lambda .}$ are DHHFLEs $(\cdot=P, B, N) . \quad h_{S_{o}}^{\lambda_{P P}}=\left\{S_{\phi_{l}<o_{\varphi_{l}}}^{\lambda_{P P}}\right\}$, $h_{S_{o}}^{\lambda_{B P}}=\left\{S_{\phi_{l}<o_{\varphi_{l}}}^{\lambda_{B P}}\right\}$, and $h_{S_{o}}^{\lambda_{N P}}=\left\{S_{\phi_{l}<o_{\varphi_{l}}}^{\lambda_{N P}}\right\}$ represent the loss degrees with DHHFLEs caused by taking actions of $a_{P}, a_{B}$, and $a_{N}$, respectively, when the object belongs to the state $X$. Analogously, $\quad h_{S_{o}}^{\lambda_{P N}}=\left\{S_{\phi_{l}<o_{\varphi_{l}}}^{\lambda_{P_{N}}}\right\}$, $h_{S_{o}}^{\lambda_{B N}}=\left\{S_{\phi_{l}<o_{\varphi_{l}}}^{\lambda_{B N}}\right\}$, and $h_{S_{o}}^{\lambda_{N N}}=\left\{S_{\phi_{l}<o_{\varphi_{l}}}^{\lambda_{N N}}\right\}$ represent the loss degrees caused by taking the same actions when the object belongs to the state $X^{c}$. Thus, in this case, $h_{S_{o}}^{\lambda} \neq \emptyset$. Based on the property of DHHFLEs and the semantics of DTRS [10, 14], we can attain a reasonable relationship as follows:

$$
\begin{aligned}
& h_{S_{o}}^{\lambda_{P P}} \preceq h_{S_{o}}^{\lambda_{B P}} \prec h_{S_{o}}^{\lambda_{N P}} \\
& h_{S_{o}}^{\lambda_{N N}} \preceq h_{S_{o}}^{\lambda_{B N}} \prec h_{S_{o}}^{\lambda_{P N}}
\end{aligned}
$$

That is the loss degree of wrong judgment is more than the loss degree of delaying decision, and both of these loss degree are more than the loss degree of correct judgment. Based on the Bayesian decision procedure of Refs. $[28,29]$, the conditional probability is one of the important elements. $\operatorname{Pr}(X \mid x i)$ is the conditional probability of an object $x$ belonging to $X$. The conditional probability of the object belonging to $X^{c}$ is $\operatorname{Pr}\left(X^{c} \mid x_{i}\right)$, they are all real numbers and satisfy $\operatorname{Pr}\left(X \mid x_{i}\right)+\operatorname{Pr}\left(X^{c} \mid x_{i}\right)=1$. Then, given an object $\mathrm{x}$, we can calculate the expected losses $R\left(a . \mid x_{i}\right)(\cdot=P, B, N)$ under the corresponding actions as follows:

$$
\begin{aligned}
& R\left(a_{P} \mid x_{i}\right)=F\left(\operatorname{le}\left(h_{S_{o}}^{\lambda_{P P}}\right)\right) \operatorname{Pr}\left(X \mid x_{i}\right)+F\left(\operatorname{le}\left(h_{S_{o}}^{\lambda_{P_{N}}}\right)\right) \operatorname{Pr}\left(X^{c} \mid x_{i}\right) \\
& R\left(a_{B} \mid x_{i}\right)=F\left(\operatorname{le}\left(h_{S_{o}}^{\lambda_{B P}}\right)\right) \operatorname{Pr}\left(X \mid x_{i}\right)+F\left(\operatorname{le}\left(h_{S_{o}}^{\lambda_{B N}}\right)\right) \operatorname{Pr}\left(X^{c} \mid x_{i}\right) \\
& R\left(a_{N} \mid x_{i}\right)=F\left(l e\left(h_{S_{o}}^{\lambda_{N P}}\right)\right) \operatorname{Pr}\left(X \mid x_{i}\right)+F\left(\operatorname{le}\left(h_{S_{o}}^{\lambda_{N N}}\right)\right) \operatorname{Pr}\left(X^{c} \mid x_{i}\right)
\end{aligned}
$$

On the basis of the results given in Refs. [5, 6], we can deduce the minimum-cost decision rules as follows:

(P) If $R\left(a_{P} \mid x_{i}\right) \leq R\left(a_{B} \mid x_{i}\right)$ and $R\left(a_{P} \mid x_{i}\right) \leq R\left(a_{N} \mid x_{i}\right)$, decide $x_{i} \in P O S(X)$;
(B) If $R\left(a_{B} \mid x_{i}\right) \leq R\left(a_{P} \mid x_{i}\right)$ and $R\left(a_{B} \mid x_{i}\right) \leq R\left(a_{N} \mid x_{i}\right)$, decide $x_{i} \in B N D(X)$; and

(N) If $R\left(a_{N} \mid x_{i}\right) \leq R\left(a_{P} \mid x\right)$ and $R\left(a_{N} \mid x_{i}\right) \leq R\left(a_{B} \mid x_{i}\right)$, decide $x_{i} \in N E G(X)$.

The positive rule(P) implies taking the action of acceptance, i.e., $x_{i} \in \operatorname{POS}(X)$. The boundary rule (B) implies taking the action of delaying the decision, i.e., $x_{i} \in B N D(X)$, and the negative rule $(\mathrm{N})$ implies taking the action of rejection, i.e., $x_{i} \in N E G(X)$.

In light of the decision-making rules [30] in DTRS model, we substitute the expression (11)-(13) of expected loss $R\left(a . \mid x_{i}\right)(\cdot=P, B, N)$ into the decision $\operatorname{rule}(P)-(N)$, and transform the inequality into the form of conditional probability and threshold comparison by deformation. The following decision-making rules in the new model can be deduced as follows:

$\left(P_{1}\right) \quad$ If $\quad \operatorname{Pr}\left(X \mid x_{i}\right) \geq \alpha \quad$ and $\quad \operatorname{Pr}\left(X \mid x_{i}\right) \geq \gamma, \quad$ decide $x_{i} \in \operatorname{POS}(X)$;

$\left(B_{1}\right)$ If $\beta \leq \operatorname{Pr}\left(X \mid x_{i}\right) \leq \alpha$, decide $x_{i} \in B N D(X)$; and

$\left(N_{1}\right) \quad$ If $\quad \operatorname{Pr}\left(X \mid x_{i}\right) \leq \beta \quad$ and $\operatorname{Pr}\left(X \mid x_{i}\right) \leq \gamma, \quad$ decide $x_{i} \in \operatorname{NEG}(X)$.

Among them, the thresholds $\alpha, \beta, \gamma$ are calculated by the loss functions $h_{S_{o}}^{\lambda},(\cdot=P, B, N)$ as follows:

$$
\alpha=\frac{\left(F\left(l e\left(h_{S_{o}}^{\lambda_{P N}}\right)\right)-F\left(l e\left(h_{S_{o}}^{\lambda_{B N}}\right)\right)\right.}{\left(\left(F\left(l e\left(h_{S_{o}}^{\lambda_{P N}}\right)\right)-F\left(l e\left(h_{S_{o}}^{\lambda_{B N}}\right)\right)\right)+\left(F\left(l e\left(h_{S_{o}}^{\lambda_{B P}}\right)\right)-F\left(l e\left(h_{S_{o}}^{\lambda_{P P}}\right)\right)\right)\right.},
$$

$$
\beta=\frac{\left(F\left(l e\left(h_{S_{o}}^{\lambda_{B N}}\right)\right)-F\left(l e\left(h_{S_{o}}^{\lambda_{N N}}\right)\right)\right.}{\left(\left(F\left(l e\left(h_{S_{o}}^{\lambda_{B N}}\right)\right)-F\left(l e\left(h_{S_{o}}^{\lambda_{N N}}\right)\right)\right)+\left(F\left(l e\left(h_{S_{o}}^{\lambda_{N P}}\right)\right)-F\left(l e\left(h_{S_{o}}^{\lambda_{B P}}\right)\right)\right)\right.},
$$

$$
\gamma=\frac{\left(F\left(l e\left(h_{S_{o}}^{\lambda_{P N}}\right)\right)-F\left(l e\left(h_{S_{o}}^{\lambda_{N N}}\right)\right)\right.}{\left(\left(F\left(l e\left(h_{S_{o}}^{\lambda_{P N}}\right)\right)-F\left(l e\left(h_{S_{o}}^{\lambda_{N N}}\right)\right)\right)+\left(F\left(l e\left(h_{S_{o}}^{\lambda_{N P}}\right)\right)-F\left(l e\left(h_{S_{o}}^{\lambda_{P P}}\right)\right)\right)\right.} .
$$

Actually, the decision rules $\left(P_{1}\right)-\left(N_{1}\right)$ are equivalent to the decision rules $(P)-(N)$. If loss functions satisfy the condition: $\quad\left(F\left(l e\left(h_{S_{o}}^{\lambda_{P N}}\right)\right)-\quad F\left(l e\left(h_{S_{o}}^{\lambda_{B N}}\right)\right)\right)\left(F\left(l e\left(h_{S_{o}}^{\lambda_{N P}}\right)\right)\right.$ $-F\left(l e\left(h_{S_{o}}^{\left.\lambda_{B P}\right)}\right)\right)>\left(F\left(l e\left(h_{S_{o}}^{\lambda_{B P}}\right)\right)-F\left(\operatorname{le}\left(h_{S_{o}}^{\lambda_{P P}}\right)\right)\right)\left(F\left(l e\left(h_{S_{o}}^{\lambda_{B N}}\right)\right)-\right.$ $\left.F\left(l e\left(h_{S_{o}}^{\lambda_{N N}}\right)\right)\right)$, then $\alpha \geq \gamma \geq \beta$ which makes $\gamma$ obsolete. Hence, the decision-making rules can be further simplified:

$\left(P_{2}\right)$ If $\operatorname{Pr}\left(X \mid x_{i}\right) \geq \alpha$, decide $x_{i} \in \operatorname{POS}(X)$;

$\left(B_{2}\right)$ If $\beta<\operatorname{Pr}\left(X \mid x_{i}\right)<\alpha$, decide $x_{i} \in B N D(X)$; and

$\left(N_{2}\right)$ If $\operatorname{Pr}\left(X \mid x_{i}\right) \leq \beta$, decide $x_{i} \in N E G(X)$.

To better explain the impact and practical significance of the thresholds $\alpha, \beta$, a simple example is listed as follows:

Example 3.1 A college want to hire a group of teachers, and there are six candidates $X=\left\{x_{1}, x_{2}, \ldots, x_{6}\right\}$ for interview. According to the comprehensive assessment of professional ability, scientific research ability, foreign language proficiency, and other attributes, the conditional 
probabilities of their achieving excellence state $(X)$ are 0.81 , $0.72,0.64,0.62,0.51,0.43$, respectively. Then the threshold $\alpha$ as the lower limit of recruitment determines how many people will be admitted. If the threshold $\alpha$ is larger, that is, the lower limit of employment is higher, then fewer people are hired. $\operatorname{POS}(X)=\left\{x_{i} \mid \operatorname{Pr}\left(X \mid x_{i}\right) \geq \alpha\right\}$; The threshold $\beta$ as the upper limit of elimination determines how many people will be eliminated. If the threshold $\beta$ is larger, that is, the higher the upper limit of elimination, then the more people will be eliminated.NEG $(X)=\left\{x_{i} \mid \operatorname{Pr}\left(X \mid x_{i}\right) \leq \beta\right\}$; Candidates who fall between the lower limit of recruitment $\alpha$ and the upper limit of elimination $\beta$ need to be further examined before making a decision. $N E G(X)=\left\{x_{i} \mid \beta<\operatorname{Pr}\left(X \mid x_{i}\right)<\alpha\right\}$. When college recruiters set standards $\alpha=0.7, \beta=0.6$, then $\operatorname{POS}(X)=\left\{x_{1}\right.$,

$\left.x_{2}\right\}, N E G(X)=\left\{x_{5}, x_{6}\right\}, B N D(X)=\left\{x_{3}, x_{4}\right\}$. That is, college recruiters decide to admit candidates $x_{1}, x_{2}$ and reject candidates $x_{5}, x_{6}$, while candidates $x_{3}, x_{4}$ needs further investigation before making a decision.

\section{The Calculation of Conditional Probability in DHHFLT Information Systems}

From the decision-making rules $P_{1}-N_{1}$, it is understood that there are two important ingredients in the TWDs: the evaluation of loss functions and the calculation of conditional probability. In Sect. 3, a novel DTRS model with DHHFLT expression of loss functions has been constructed. As for the calculation of the conditional probability, it depends on DHHFLT information systems. Thus, we need to define the DHHFLT information systems firstly. Given that $A T$ is a finite set of conditional attributes, $V=\bigcup_{a \in A T} V_{a}, \quad V_{a} \quad$ is a domain of attribute a. $g: U \times A T \rightarrow V$ is a function such that $g\left(x_{i}, a\right) \in V_{a}$ for every $a \in A T, x_{i} \in U$, where $g\left(x_{i}, a\right)$ is a DHHFLT. $U=\left\{x_{1}, x_{2}, \ldots, x_{m}\right\}$ denotes a discrete set of $\mathrm{m}$ feasible objects, $A T=\left\{c_{1}, c_{2}, \ldots, c_{n}\right\}$ denotes the attribute set of the DHHFLT information systems. $\omega=\left(\omega_{1}, \omega_{2}, \ldots, \omega_{n}\right)^{T}$ is the weight vector of all attributes, it satisfies $0 \leq \omega_{j} \leq 1$ and $\sum_{j=1}^{n} \omega_{j}=1$. The evaluation of the object $x_{i}$ regarding the attribute $c_{j}$ is denoted as $g\left(x_{i}, c_{j}\right)=h_{S_{o}}^{i j}(i=1,2, \ldots$, $m ; j=1,2, \ldots, n)$.

For instance, there are four cars $x_{1}-x_{4}$ for customers to choose from, four attributes are determined as follows: (1) $c_{1}$ price level; (2) $c_{2}$ comfort level; (3) $c_{3}$ safety level; (4) $c_{4}$ velocity level. Among them, $c_{1}$ belongs to cost attribute, the others belong to profit attribute. After determining the attributes, we need to collect the evaluation information with DHHFLT. Next, we need to determine the weight of each attribute by entropy weight method [31], specific steps are as follows:

(1) According to the formulas (2) and (8), the evaluation value with DHHFLT is transformed to a real number. Then, we can obtain a normalized information matrix $H=\left[h_{i j}\right]_{m \times n}$, where $h_{i j}=F\left(l e\left(h_{S_{o}}^{i j}\right)\right)$.

(2) Calculates the proportion of the attribute value of the ith object under the attribute $c_{j}$ in the normalized information matrix $H=\left[h_{i j}\right]_{m \times n}$.

$p_{i j}=\frac{h_{i j}}{\sum_{i=1}^{m} h_{i j}},(j \in n)$

Through this step, we can eliminate the influence of dimension and control the attribute value within the interval $[0,1]$.

(3) Calculates the entropy of the attribute $c_{j}$ :

$Q_{j}=-\frac{1}{\ln m} \sum_{i=1}^{m} p_{i j} \ln p_{i j},(j \in n)$

Assume $p_{i j} \ln p_{i j}=0$ when $p_{i j}=0 . Q_{j}$ represents the uncertainty of information in attribute $\mathrm{j}$.

(4) Calculates the difference coefficient of the attribute $c_{j}$ :

$\theta_{j}=1-Q_{j}$

$\theta_{j}$ represents the certainty of information in attribute j.

(5) Determines the weight of conditional attributes. The formula for calculating the weight of the $j t h$ attribute is

$\omega_{j}=\frac{\theta_{j}}{n-\sum_{j=1}^{n} Q_{j}}$

Then, we can get the weight of each attribute by entropy weight method $\omega=\left(\omega_{1}, \omega_{2}, \ldots, \omega_{n}\right)^{T}$. In other words, the higher the certainty of the attribute value, the greater the weight assigned to the attribute.

Next, we need to convert the cost attribute to the profit attribute as follows:

$\widetilde{h}_{i j}=1-h_{i j}(i=1,2, \ldots, m ; j=1,2, \ldots, n)$

Then, we can obtain the attribute normalized information matrix $\widetilde{H}=\left[\widetilde{h}_{i j}\right](i=1,2, \ldots, m ; j=1,2, \ldots, n)$. If all the attributes belong to profit attribute, we do not have to deal with it.

At last, we can calculate the conditional probability by aggregating the attribute information by weight as follows: 


$$
\operatorname{Pr}\left(X \mid x_{i}\right)=\sum_{j=1}^{n} \omega_{j} \widetilde{h}_{i j}(i=1,2, \ldots, m ; j=1,2, \ldots, n),
$$

where $\operatorname{Pr}\left(X \mid x_{i}\right) \in[0,1]$, the greater the profit attribute value of an object, the higher the conditional probability that it belongs to state $\mathrm{X}$.

\section{The Process of TWDs}

By introducing the results mentioned above, a novel method of TWDs in the DHHFLT environment is proposed by us. The specific steps of method proposed are shown as follows:

Step 1: Based on the practical context, we determine the definition of DHHFLT information system $I S=(U, A T, V, g)$, including objects and conditional attributes. Then, we collect the outcomes of IS, and we categorize objects and construct the loss function matrixes of Table 1 accordingly.

Step 2: We determine the weight of each attribute by entropy weight method based on the outcomes of IS.

Step 3: We check the conditional attributes of IS, if all the attributes belong to profit attribute, go to Step 5.

Step 4: We need to convert the cost attribute to the profit attribute based on the formula (21).

Step 5: We can calculate the conditional probability by the formula (22).

Step 6: Based on the loss functions matrixes, the thresholds $\alpha, \beta, \gamma$ can be calculated, respectively, by the formulas (14) - (16).

Step 7: In the light of the decision rules $P_{1}-N_{1}$, we can ascertain the decision result for each object further.

For the sake of clarity, the process of the method proposed by us is shown in Fig. 1.

\section{A Case Study About the Problem of Work Resumption After Epidemic}

At the beginning of 2020, almost all enterprises and individual businesses in China stopped production and business for more than a month, which caused about 2.5 trillion yuan of direct losses to economic activities. At present, as the epidemic prevention and control situation in China continues to improve, the resumption of work and production has been put on the agenda, which is of great significance to the recovery of economic development. There are seven companies facing with the difficult decision about whether the enterprise should resume work and production immediately. They are $x_{1}$ : restaurant; $x_{2}$ : logistics company; $x_{3}$ : amusement park; $x_{4}$ : hotel; $x_{5}$ : building materials factory; $x_{6}$ : clothing store; $x_{7}$ : parts factory, respectively. Before making decisions, these enterprises need to estimate the probability of virus infection within the enterprise if they immediately resume work and production. Thus, in the structure of TWDs, the set of the states $\Omega=\left\{X, X^{c}\right\}$ indicates that this enterprise will be safe or infected after resuming work and production. Because the calculation of the conditional probability depends on DHHFLT information systems, we determine the conditional attribute in the information system as follows: $c_{1}$ : the frequency of contact with people; $c_{2}$ : the rigor of preventive measures; $c_{3}$ : the level of infection in the surrounding environment. In this section, we apply the method of Sect. 5 to assist the research and make decisions for these enterprises.

Step 1: We need to determine the definition of DHHFLT information system $I S=(U, A T, V, g)$. In summary, we can obtain: $U=\left\{x_{1}, x_{2}, \ldots, x_{7}\right\}$ and $A T=c_{1}, c_{2}, c_{3}$. All the evaluation will be provided based on the following two LTSs by the experts:

$$
\begin{aligned}
& S=\left\{s_{-4}=\text { extremelylow }, s_{-3}=\text { velylow },\right. \\
& s_{-2}=\text { low }, s_{-1}=\text { slightlylow }, s_{0}=\text { medium }, \\
& s_{1}=\text { slightlyhigh, } s_{2}=\text { high, } s_{3}=\text { veryhigh, } \\
& \left.\quad s_{4}=\text { extremelyhigh }\right\} \\
& O=\left\{o_{-4}=\text { farfrom, } o_{-3}=\text { scarcely, } o_{-2}=\right.\text { onlyalittle, } \\
& o_{-1}=\text { alittle, } o_{0}=\text { justright }, \\
& o_{1}=\text { much, } o_{2}=\text { verymuch }, o_{3} \\
& \left.=\text { extremelymuch, } o_{4}=\text { entirely }\right\}
\end{aligned}
$$

Then, we collect evaluation for each object regarding the attributes $c_{1}-c_{3}$ from these enterprises's experts. Experts evaluate attributes based on their knowledge and research of the company and use DHHFLT to represent evaluation information. The corresponding DHHFLT information system is displayed in Table 2 .

For each element of Table 2, it is expressed by a DHHFLT, and it represents the evaluation of the object $x_{i}$ regarding the attribute $c_{j}(i=1,2, \ldots, 7 ; j=1,2,3)$. According to the classification criteria for large, medium, small, and micro enterprises released by the National Bureau of Statistics, these enterprises are divided into four categories in terms of operating income, number of employees, and total assets. $A_{1}$ : large enterprises; $A_{2}$ : medium enterprises; $A_{3}$ : small enterprises; $A_{4}$ : micro enterprises, where $x_{2}, x_{5} \in A_{1} ; x_{3} \in A_{2} ; x_{1}, x_{4}, x_{7} \in A_{3}$; 


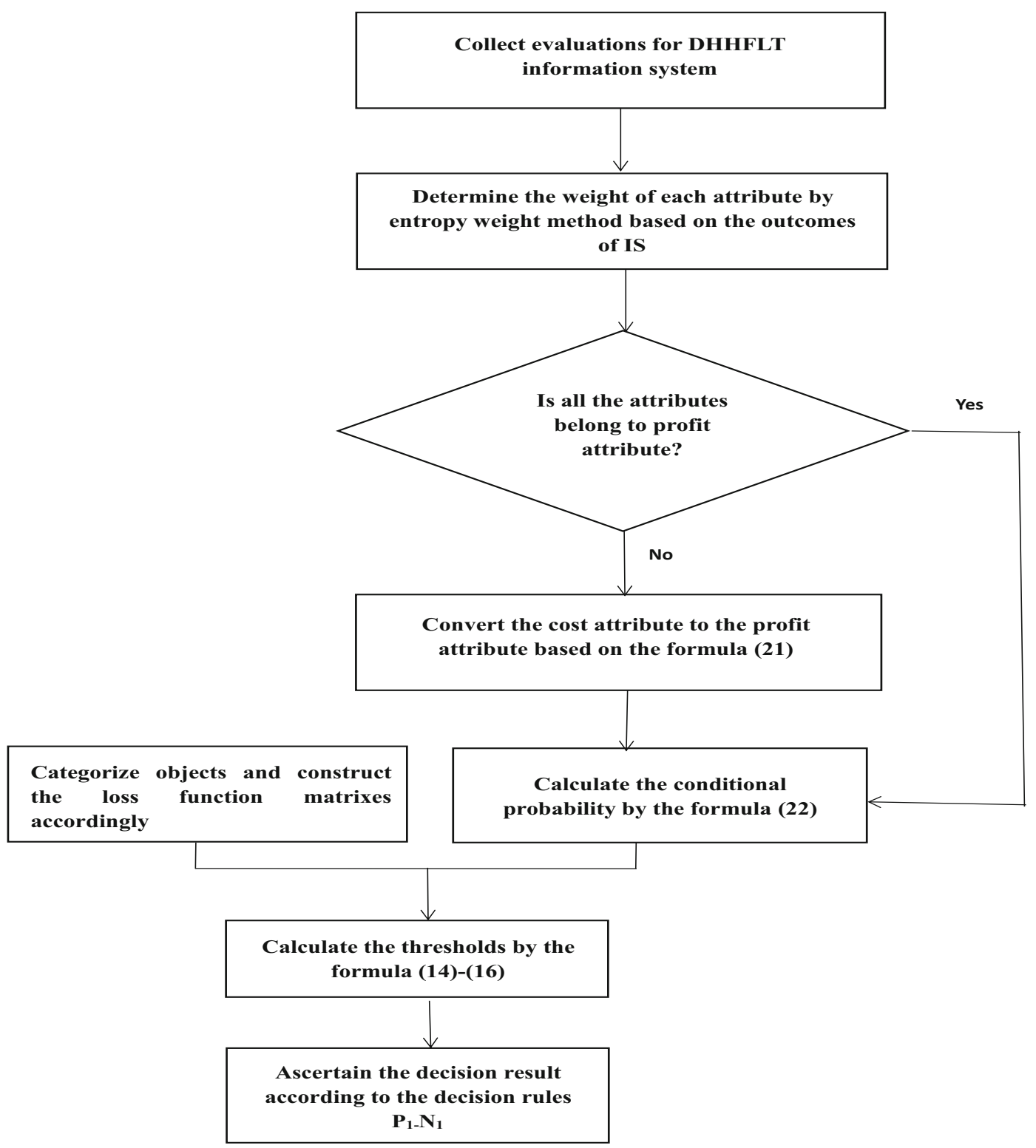

Fig. 1 The process of the method proposed by us

$x_{6} \in A_{4}$. Since enterprises of different sizes take the same action in the same state have different losses, we respectively construct different loss function matrixes for the four types of enterprises (Tables 3, 4, 5, and 6):

All the information about the loss function comes from assessments by scholars who study the laws of economic change.

Step 2: We need to determine the weight of each attribute by entropy weight method on the basis of the outcomes of IS. We can calculate the weight of each attribute according to the special steps in Sect. 4.

(1)-(2) According to the formulas (2) and (8), the evaluation value of Table 2 is transformed to a real number. Then, we can obtain a normalized information matrix in Table 7.
(3) Based on formula (18), we calculate the entropy of each attribute:

$Q_{1}=0.9576, Q_{2}=0.9759, Q_{3}=0.9377$

(4)-(5) Based on the formulas (19)-(20), we calculate the weight of each attribute:

$\omega_{1}=0.3292, \omega_{2}=0.1871, \omega_{3}=0.4837$

Step 3: We check the conditional attributes of IS, the attributes $c_{1}, c_{3}$ belong to cost attribute, the attributes $c_{2}$ belong to profit attribute.

Steps 4-5: We convert the cost attribute to the profit attribute based on the formula (21). And then we can calculate the conditional probability of these enterprises will be safe after resuming work and production by the formula (22). The results are displayed in Table 8 . 
Step 6: In light of the loss functions matrixes, the thresholds $\alpha, \beta, \gamma$ can be calculated, respectively, by the formulas (14)-(16). The results of calculation are shown in Table 9 .

Because there is a relationship between the threshold we obtained: $\alpha>\gamma>\beta$, we just need to concern about the values of $\alpha$ and $\beta$.

Step 7: According to the decision rules $P_{1}-N_{1}$, we can ascertain the decision result for each object. The results are shown in Fig. 2. $\operatorname{POS}(X)=\left\{x_{4}, x_{5}\right\} ; B N D(X)=\left\{x_{2}, x_{7}\right\}$; $N E G(X)=\left\{x_{1}, x_{3}, x_{6}\right\}$. Due to the small scale of the $x_{4}$ : hotel, its frequency of contact with people is slightly low, and the level of infection in the surrounding environment is very low, the probability of infection will be low when they returning to work, so they can return to work immediately. $x_{5}$ : Building materials factory's scale is large, once they stop working, the loss is huge, and its rigor of preventive measures is very high. Thus, it can also resume work immediately. However, $x_{1}$ : restaurant, $x_{3}$ : amusement park, and $x_{6}$ : clothing store have a large daily traffic flow and a high probability of infection, so it is suggested that they stop working, and $x_{2}$ : logistics companies, $x_{7}$ : parts factory need to gather more information and wait for further decisions.

\section{Comparison and Discussion}

Next, we will compare the method proposed by us with the other method and discuss the advantages and limitations of our proposed method.

\subsection{Comparison Analyses}

The TWDs method proposed by Sun et al. [32] can only deal with the information of linguistic term set. In order to facilitate comparison, the DHFLEs can be transformed into hesitant fuzzy linguistic elements (HFLEs) by expurgating the second hierarchy linguistic information. Then we can transform Table 2 into Table 10.
Table 3 Loss function information with DHHFLT for $A_{1}$

\begin{tabular}{lll}
\hline & $\mathrm{X}(\mathrm{P})$ & $X^{c}(N)$ \\
\hline$a_{P}$ & $\left\{s_{3}, s_{3\left\langle o_{2}\right\rangle}\right\}$ & $\left\{s_{-4}\right\}$ \\
$a_{B}$ & $\left\{s_{-1\left\langle o_{-1}\right\rangle}\right\}$ & $\left\{s_{1\left\langle o_{-2}\right\rangle},\left\{s_{1}\right\}\right\}$ \\
$a_{N}$ & $\left\{s_{-2\left\langle o_{-3}\right\rangle}\right\}$ & $\left\{s_{3\left\langle o_{-1}\right\rangle}\right\}$ \\
\hline
\end{tabular}

Table 4 Loss function information with DHHFLT for $A_{2}$

\begin{tabular}{lll}
\hline & $\mathrm{X}(\mathrm{P})$ & $X^{c}(N)$ \\
\hline$a_{P}$ & $\left\{s_{3\left\langle o_{-3}\right\rangle}, s_{3\left\langle o_{-1}\right\rangle}\right\}$ & $\left\{s_{-4}\right\}$ \\
$a_{B}$ & $\left\{s_{-1\left\langle o_{-}\right\rangle}\right\}$ & $\left\{s_{0\left\langle o_{-1}\right\rangle}\right\}$ \\
$a_{N}$ & $\left\{s_{-3\left\langle o_{2}\right\rangle}\right\}$ & $\left\{s_{2\left\langle o_{3}\right\rangle}, s_{3\left\langle o_{-3}\right\rangle}\right\}$ \\
\hline
\end{tabular}

Table 5 Loss function information with DHHFLT for $A_{3}$

\begin{tabular}{lll}
\hline & $\mathrm{X}(\mathrm{P})$ & $X^{c}(N)$ \\
\hline$a_{P}$ & $\left\{s_{2\left\langle o_{3}\right\rangle}\right\}$ & $\left\{s_{-4}\right\}$ \\
$a_{B}$ & $\left\{s_{-1\left\langle o_{-3}\right\rangle}\right\}$ & $\left\{s_{0\left\langle o_{-2}\right\rangle}, s_{0\left\langle o_{-3}\right\rangle}\right\}$ \\
$a_{N}$ & $\left\{s_{-3}, s_{-3\left\langle o_{-2}\right\rangle}\right\}$ & $\left\{s_{2\left\langle o_{2}\right\rangle}\right\}$ \\
\hline
\end{tabular}

Table 6 Loss function information with DHHFLT for $A_{4}$

\begin{tabular}{lll}
\hline & $\mathrm{X}(\mathrm{P})$ & $X^{c}(N)$ \\
\hline$a_{P}$ & $\left\{s_{2\left\langle o_{2}\right\rangle}\right\}$ & $\left\{s_{-4}\right\}$ \\
$a_{B}$ & $\left\{s_{-1\left\langle o_{1}\right\rangle}, s_{-1\left\langle o_{3}\right\rangle}\right\}$ & $\left\{s_{1\left\langle o_{-2}\right\rangle}\right\}$ \\
$a_{N}$ & $\left\{s_{-3\left\langle o_{-3}\right\rangle}\right\}$ & $\left\{s_{2\left\langle o_{1}\right\rangle}\right\}$ \\
\hline
\end{tabular}

Since the TWDs method proposed by Sun et al. [32] does not classify the objects, we assume that the objects are of the $A_{3}$ category. Then, the loss functions for $A_{3}$ in Table 5 are transformed into Table 11.

Then, the results of conditional probability are shown in Table 12.
Table 2 The corresponding DHHFLT information system

\begin{tabular}{llll}
\hline & $c_{1}$ & $c_{2}$ & $c_{3}$ \\
\hline$x_{1}$ & $\left\{s_{3}, s_{3\left\langle o_{2}\right\rangle}\right\}$ & $\left\{s_{1\left\langle o_{-1}\right\rangle}, s_{1\left\langle o_{2}\right\rangle}, s_{2\left\langle o_{-3}\right\rangle}\right\}$ & $\left\{s_{2\left\langle o_{-2}\right\rangle}, s_{2\left\langle o_{1}\right\rangle}\right\}$ \\
$x_{2}$ & $\left\{s_{1\left\langle o_{1}\right\rangle}, s_{1\left\langle o_{3}\right\rangle}\right\}$ & $\left\{s_{3}, s_{3\left\langle o_{2}\right\rangle}\right\}$ & $\left\{s_{-1\left\langle o_{2}\right\rangle}, s_{0\left\langle o_{-3}\right\rangle}, s_{0\left\langle o_{-1}\right\rangle}\right\}$ \\
$x_{3}$ & $\left\{s_{2\left\langle o_{3}\right\rangle}, s_{3\left\langle o_{-1}\right\rangle}, s_{3\left\langle o_{2}\right\rangle}\right\}$ & $\left\{s_{-1\left\langle o_{2}\right\rangle}, s_{0}, s_{0\left\langle o_{3}\right\rangle}\right\}$ & $\left\{s_{1\left\langle o_{3}\right\rangle}, s_{2\left\langle o_{1}\right\rangle}\right\}$ \\
$x_{4}$ & $\left\{s_{-1\left\langle o_{2}\right\rangle}, s_{0\left\langle o_{-1}\right\rangle}\right\}$ & $\left\{s_{2\left\langle o_{1}\right\rangle}, s_{2\left\langle o_{3}\right\rangle}, s_{3\left\langle o_{-2}\right\rangle}\right\}$ & $\left\{s_{-3\left\langle o_{1}\right\rangle}, s_{-2\left\langle o_{-}\right\rangle}\right\}$ \\
$x_{5}$ & $\left\{s_{-2\left\langle o_{-3}\right\rangle}, s_{-2\left\langle o_{-1}\right\rangle}\right\}$ & $\left\{s_{2\left\langle o_{3}\right\rangle}, s_{3}, s_{3\left\langle o_{1}\right\rangle}\right\}$ & $\left\{s_{-3}, s_{-2\left\langle o_{1}\right\rangle}\right\}$ \\
$x_{6}$ & $\left\{s_{2\left\langle o_{2}\right\rangle}, s_{3}, s_{3\left\langle o_{1}\right\rangle}\right\}$ & $\left\{s_{-2\left\langle o_{3}\right\rangle}, s_{-1\left\langle o_{-2}\right\rangle}\right\}$ & $\left\{s_{1\left\langle o_{3}\right\rangle}, s_{2\left\langle o_{1}\right\rangle}\right\}$ \\
$x_{7}$ & $\left\{s_{1\left\langle o_{-3}\right\rangle}, s_{1}, s_{1\left\langle o_{1}\right\rangle}\right\}$ & $\left\{s_{0\left\langle o_{1}\right\rangle}, s_{0\left\langle o_{3}\right\rangle}\right\}$ & $\left\{s_{-1\left\langle o_{1}\right\rangle}, s_{-1\left\langle o_{3}\right\rangle}\right\}$ \\
\hline
\end{tabular}


Table 7 The normalized information matrix

Table 8 The normalized attributes information matrix and conditional probability

\begin{tabular}{lllllll}
\hline & $c_{1}$ & $c_{2}$ & $c_{3}$ & $p_{1}$ & $p_{2}$ & $p_{3}$ \\
\hline$x_{1}$ & $\frac{29}{32}$ & $\frac{31}{48}$ & $\frac{47}{64}$ & $\frac{58}{293}$ & $\frac{124}{891}$ & $\frac{47}{223}$ \\
$x_{2}$ & $\frac{11}{16}$ & $\frac{29}{32}$ & $\frac{7}{16}$ & $\frac{44}{293}$ & $\frac{58}{297}$ & $\frac{28}{223}$ \\
$x_{3}$ & $\frac{7}{8}$ & $\frac{49}{96}$ & $\frac{3}{4}$ & $\frac{56}{293}$ & $\frac{98}{891}$ & $\frac{48}{223}$ \\
$x_{4}$ & $\frac{29}{64}$ & $\frac{13}{16}$ & $\frac{11}{64}$ & $\frac{29}{293}$ & $\frac{52}{297}$ & $\frac{11}{223}$ \\
$x_{5}$ & $\frac{3}{16}$ & $\frac{7}{8}$ & $\frac{13}{64}$ & $\frac{12}{293}$ & $\frac{56}{297}$ & $\frac{13}{223}$ \\
$x_{6}$ & $\frac{83}{96}$ & $\frac{21}{64}$ & $\frac{3}{4}$ & $\frac{166}{879}$ & $\frac{7}{99}$ & $\frac{48}{223}$ \\
$x_{7}$ & $\frac{29}{48}$ & $\frac{9}{16}$ & $\frac{7}{16}$ & $\frac{116}{879}$ & $\frac{4}{33}$ & $\frac{28}{223}$ \\
\hline
\end{tabular}

\begin{tabular}{ccccc}
\hline & $c_{1}$ & $c_{2}$ & $c_{3}$ & $\operatorname{Pr}\left(X \mid x_{i}\right)$ \\
\hline$x_{1}$ & $\frac{3}{32}$ & $\frac{31}{48}$ & $\frac{17}{64}$ & 0.2802 \\
$x_{2}$ & $\frac{5}{16}$ & $\frac{29}{32}$ & $\frac{9}{16}$ & 0.5445 \\
$x_{3}$ & $\frac{1}{8}$ & $\frac{49}{96}$ & $\frac{1}{4}$ & 0.2576 \\
$x_{4}$ & $\frac{35}{64}$ & $\frac{13}{16}$ & $\frac{53}{64}$ & 0.7326 \\
$x_{5}$ & $\frac{13}{16}$ & $\frac{7}{8}$ & $\frac{51}{64}$ & 0.8166 \\
$x_{6}$ & $\frac{13}{96}$ & $\frac{21}{64}$ & $\frac{1}{4}$ & 0.2269 \\
$x_{7}$ & $\frac{19}{48}$ & $\frac{9}{16}$ & $\frac{9}{16}$ & 0.5076 \\
\hline
\end{tabular}

On the basis of the method proposed by Sun et al. [32], the thresholds can be obtained: $\alpha=0.5714, \beta=0.5, \gamma=0.5455$. Then, we can determine the decision result for each object: $\operatorname{POS}(X)=\left\{x_{2}, x_{4}, x_{5}\right\}$; $B N D(X)=\left\{x_{7}\right\} ; N E G(X)=\left\{x_{1}, x_{3}, x_{6}\right\}$.

From Table 13, we can find that the values of $\beta, \gamma$ are increasing obviously attained by Sun's method. The results of our decisions are almost the same, and the difference between our results is the object $x_{2}$ is classified into $\operatorname{POS}(X)$ from $B N D(X)$. The reasons can be concluded as follows:

(1) Due to the transformation of DHFLEs to HFLEs, we lose a part of original linguistic information.

(2) the three-way decisions method proposed by Sun et al. [32] does not construct the corresponding loss function matrix according to the different object categories.

Therefore, we can get that the DHHFLT information can describe the original linguistic information more accurately. Besides, it is necessary to construct the corresponding loss function matrix according to the different object categories.

\subsection{Discussions on the Advantages and Limitations}

DTRSs model, as one of the elements of the three-way decisions method, is mainly responsible for displaying the loss caused by the actions taken by the object in different states, and selecting the action with the minimum loss. The conditional probability is the another element of the TWDs method. In the most of the existing TWDs models [33, 34], the conditional probability is given directly which makes the decision result seem not so rigorous. We get the weight of each attribute by entropy weight method, and take the weighted aggregation of attribute information as the conditional probability. Moreover, we construct the corresponding loss function matrix according to the different object categories. It makes the results of decisions more objective and reasonable.

As a kind of linguistic term, DHHFLTS conforms to people's expression habits. In contrast to single linguistic term set, DHHFLTSs provide more flexible manner to express qualitative information by ways of linguistic expressions. Our proposed method is constructed under the DHHFLT environment. DHHFLT was mostly applied in the multi-attribute decision-making methods $[25,26]$ previously, the TWDs model on the basis of the DHHFLT information systems is a new research content. It has high research value.

The main advantages of the proposed method are displayed as follows:

Fig. 2 Three-way decisions based on different $\operatorname{Pr}\left(X \mid x_{i}\right)$ 
Table 10 The corresponding HFLEs information system

\begin{tabular}{llll}
\hline & $c_{1}$ & $c_{2}$ & $c_{3}$ \\
\hline$x_{1}$ & $\left\{s_{3}\right\}$ & $\left\{s_{1}, s_{2}\right\}$ & $\left\{s_{2}\right\}$ \\
$x_{2}$ & $\left\{s_{1}\right\}$ & $\left\{s_{3}\right\}$ & $\left\{s_{-1}, s_{0}\right\}$ \\
$x_{3}$ & $\left\{s_{2}, s_{3}\right\}$ & $\left\{s_{-1}, s_{0}\right\}$ & $\left\{s_{1}, s_{2}\right\}$ \\
$x_{4}$ & $\left\{s_{-1}, s_{0}\right\}$ & $\left\{s_{2}, s_{3}\right\}$ & $\left\{s_{-3}, s_{-2}\right\}$ \\
$x_{5}$ & $\left\{s_{-2}\right\}$ & $\left\{s_{2}, s_{3}\right\}$ & $\left\{s_{-3}, s_{-2}\right\}$ \\
$x_{6}$ & $\left\{s_{2}, s_{3}\right\}$ & $\left\{s_{-2}, s_{-1}\right\}$ & $\left\{s_{1}, s_{2}\right\}$ \\
$x_{7}$ & $\left\{s_{1}\right\}$ & $\left\{s_{0}\right\}$ & $\left\{s_{-1}\right\}$ \\
\hline
\end{tabular}

Table 11 Loss function information with HFLEs for $A_{3}$

\begin{tabular}{lll}
\hline & $\mathrm{X}(\mathrm{P})$ & $X^{c}(N)$ \\
\hline$a_{P}$ & $\left\{s_{2}\right\}$ & $\left\{s_{-4}\right\}$ \\
$a_{B}$ & $\left\{s_{-1}\right\}$ & $\left\{s_{0}\right\}$ \\
$a_{B}$ & $\left\{s_{-3}\right\}$ & $\left\{s_{2}\right\}$ \\
\hline
\end{tabular}

(1) We apply the TWDs method to the decision-making of work resumption after the epidemic. The method meets the decision-making needs of enterprises, and the decision results will minimize the losses caused by the epidemic to enterprises. The research also expands the application field of the TWDs method. Then, we propose a conditional probability calculation method based on DHHFLT information system, which makes the decision result more objective and reasonable.

(2) As a kind of convenient and effective tool, it is used to describe the loss functions in TWDs and the attributes information of objects, which let the experts make decisions more easily. The experts can use it intuitively to give the evaluation value, which saves a lot of time for decision-making.

However, there are some limitations with the proposed method:

(1) For convenience of calculations, this article does not discuss group decisions, nor does it consider the weights of different experts. We plan to extend this method to group decisions in future work, and make it more practical.

(2) The method proposed in this paper is applicable to the company which has not started before the
Table 13 The comparison of thresholds

\begin{tabular}{llll}
\hline $\mathrm{U}$ & $\alpha$ & $\beta$ & $\gamma$ \\
\hline method proposed by Sun & 0.5714 & 0.5 & 0.5455 \\
method proposed by us & 0.5714 & 0.3243 & 0.48 \\
\hline
\end{tabular}

decision to resume work. If an infection occurs after a company has started work, the probability of virus transmission will need to be reassessed. And then the decision in this case will require to be discussed further. This is also an interesting potential research point.

\section{Conclusions}

In this paper, we extend the TWDs with DTRSs to the double hierarchy hesitant fuzzy linguistic term environment by considering the loss functions being expressed by DHHFLTs. And we get the weight of each attribute by entropy weight method, and take the weighted aggregation of attribute information as the conditional probability. Next, according to the difference of company size, we put forward the corresponding loss function matrix, and we design the specific steps of the method proposed by us. Finally, we apply the TWDs method to the decision-making of work resumption after the epidemic. Our research provides a scientific decision-making method for all enterprises to resume work and production after the epidemic from the perspective of three-way decisions, so as to minimize the loss. Therefore, the method proposed by us has relatively high value in theory and application.
Table 12 The results of conditional probability

\begin{tabular}{llllllll}
\hline $\mathrm{U}$ & $x_{1}$ & $x_{2}$ & $x_{3}$ & $x_{4}$ & $x_{5}$ & $x_{6}$ & $x_{7}$ \\
\hline $\operatorname{Pr}\left(X \mid x_{i}\right)$ & 0.3182 & 0.5853 & 0.3084 & 0.7466 & 0.7960 & 0.2796 & 0.5302 \\
\hline
\end{tabular}


Acknowledgements This work was supported by the Natural Science Foundation of China (Nos.71771155, 71971119), and the Collaborative Innovation Center of Audit Information Engineering and Technology, Nanjing Audit University, Nanjing, China (No.18CICA01).

\section{References}

1. Ena, J., Wenzel, R.P.: A novel coronavirus emerges. Revista Clinica Espanola 220(2), 115-116 (2020)

2. Kelvin, D., Rubino, S.: Fear of the novel coronavirus. J. Infect. Dev. Count. 14(1), 1-2 (2020)

3. Khan, S., Ali, A., Siddique, R., Nabi, G.: Novel coronavirus is putting the whole world on alert. J. Hosp. Infect. 104(3), 252-253 (2020)

4. Lieke, K., Pierre, K.: Graded return-to-work as a stepping stone to full work resumption. J. Health Econ. 65, 189-209 (2019)

5. Wong, S.K.M., Lingras, P.: A decision-theoretic rough set model. In: Proceedings of the 5th International Symposium on Methodologies for Intelligent Systems. North-Holland (1990) pp. 17-24

6. Yao, Y.Y.: Three-way decisions with probabilistic rough sets. Inform. Sci. 180, 341-353 (2010)

7. Lingras, P., Chen, M., Miao, D.Q.: Rough cluster quality index based on decision theory. IEEE Trans. Knowl. Data Eng. 21(7), 1014-1026 (2008)

8. Yu, H., Liu, Z.G., Wang, G.Y.: Automatically determining the number of clusters using decision-theoretic rough set. In: International Conference on Rough Sets and Knowledge Technology. Springer, Berlin (2011), pp. 504-513

9. Li, H.X., Zhou, X.Z.: Risk decision making based on decisiontheoretic rough set: a three-way view decision model. Int. J. Comput. Intell. Syst. 4(1), 1-11 (2011)

10. Liang, D.C., Liu, D.: A novel risk decision making based on decision-theoretic rough sets under hesitant fuzzy information. IEEE Trans. Fuzzy Syst. 23(2), 237-247 (2015)

11. Maldonado, S., Peters, G., Weber, R.: Credit scoring using threeWay decisions with probabilistic rough sets. Inform. Sci. 507, 700-714 (2018)

12. Zadeh, L.A.: Fuzzy sets. Inform. Control 8(3), 338-353 (1965)

13. Liang, D.C., Liu, D., Pedrycz, W., Hu, P.: Triangular fuzzy decision-theoretic rough sets. Int. J. Approx. Reason. 54(8), 1087-1106 (2013)

14. Liang, D.C., Liu, D.: Deriving three-way decisions from intuitionistic fuzzy decision-theoretic rough sets. Inform. Sci. 300, 28-48 (2015)

15. Liang, D.C., Xu, Z.S., Liu, D.: Three-way decisions based on decision-theoretic rough sets with dual hesitant fuzzy information. Inform. Sci. 396, 127-143 (2017)

16. Liang, D.C., Pedrycz, W., Liu, D., et al.: Three-way decisions based on decision-theoretic rough sets under linguistic assessment with the aid of group decision making. Appl. Soft Comput. 29, 256-269 (2015)

17. Yang, J.L., Yao, Y.Y.: Semantics of soft sets and three-way decision with soft sets. Knowl. Based Syst. 194, 105538 (2020)

18. Ye, J., Zhan, J.M., Xu, Z.S.: A novel decision-making approach based on three-way decisions in fuzzy information systems. Inform. Sci. 541, 362-390 (2020)

19. Liang, D.C., Xu, Z.S., Liu, D., et al.: Method for three-way decisions using ideal TOPSIS solutions at Pythagorean fuzzy information. Inform. Sci. 435, 282-295 (2018)

20. Zadeh, L.A.: What is computing with words (CWW)? Stud. Fuzziness Soft Comput. 277, 3-37 (2013)
21. Rodriguez, R.M., Martinez, L., Herrera, F.: Hesitant fuzzy linguistic term sets for decision making. IEEE Trans. Fuzzy Syst. 20(1), 109-119 (2011)

22. Herrera, F., Martnez, L.: A 2-tuple fuzzy linguistic representation model for computing with words. IEEE Trans. Fuzzy Syst. 8(6), $746-752(2000)$

23. $\mathrm{Xu}, \mathrm{Z} . \mathrm{S}$.: A method based on linguistic aggregation operators for group decision making with linguistic preference relations. Inform. Sci. 166(1-4), 19-30 (2004)

24. Xu, Z.S., Wang, H.: On the syntax and semantics of virtual linguistic terms for information fusion in decision making. Inform. Fusion 34, 43-48 (2017)

25. Gou, X.J., Liao, H.C., Xu, Z.S., Herrera, F.: Double hierarchy hesitant fuzzy linguistic term set and MULTIMOORA method: a case of study to evaluate the implementation status of haze controlling measures. Inform. Fusion 38, 22-34 (2017)

26. Gou, X.J., Liao, H.C., Xu, Z.S., Herrera, F.: Multiple criteria decision making based on distance and similarity measures under double hierarchy hesitant fuzzy linguistic environment. Comput. Indust. Eng. 126, 516-530 (2018)

27. Gou, X.J., Liao, H.C., Xu, Z.S., et al.: Group decision making with double hierarchy hesitant fuzzy linguistic preference relations: consistency based measures, index and repairing algorithms and decision model. Inform. Sci. 489, 93-112 (2019)

28. Pawlak, Z.: Rough sets. Int. J. Comput. Inform Sci. 11(5), 341-356 (1982)

29. Chien, Y.: Pattern classification and scene analysis. IEEE Trans. Autom. Control 19(4), 462-463 (1974)

30. Qian, J., Liu, C.H., Miao, D.Q., Yue, X.D.: Sequential three-way decisions via multi-granularity. Inform. Sci. 507, 606-629 (2019)

31. Na, D., Sun, L., Zhang, Q., et al.: Uncertain linguistic for determining attribute weights method in multiple attribute group decision making. Sci. Technol. Vis. 2, 28-52 (2016)

32. Sun, B.Z., Ma, W.M., Li, B.J., Li, X.N.: Three-way decisions approach to multiple attribute group decision making with linguistic information-based decision-theoretic rough fuzzy set. Int. J. Approx. Reason. 93, 424-442 (2018)

33. Liu, D., Yao, Y.Y., Li, T.R.: Three-way investment decisions with decision-theoretic rough sets. Int. J. Comput. Intell. Syst. 4(1), 66-74 (2011)

34. Liu, D., Li, T.R., Liang, D.C.: Three-way government decision analysis with decision-theoretic rough sets. Int. J. Uncert. Fuzziness Knowl-Based Syst. 20(1), 119-132 (2012)

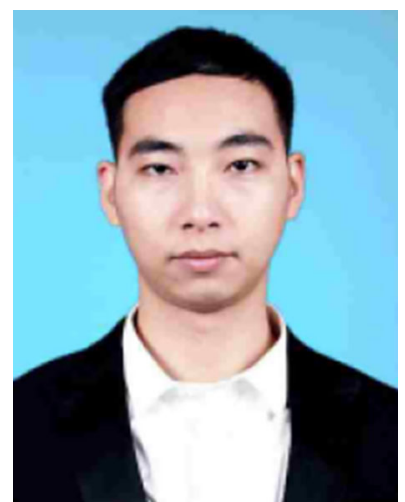

Xiang $\mathbf{L i}$ is currently working toward the doctor's degree with the School of Economics and Management, Southeast University, Nanjing, China. His research interests include three-way decisions and fuzzy decision analysis. 


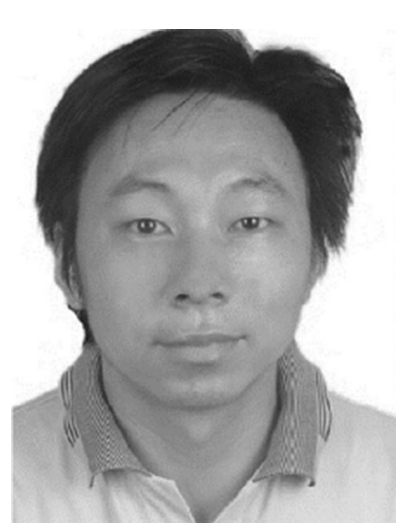

Hai Wang received the B.Sc. degree in Mathematics from China University of Geoscience in 2005, the M.Sc. degree in Management Science and Engineering form Nanjing Normal University in 2012, and the $\mathrm{Ph} . \mathrm{D}$. degree in Management Science and Engineering form Southeast University in 2017. $\mathrm{He}$ is currently a Lecturer with School of Information Engineering at Nanjing Audit University, Nanjing, China. Dr. Wang's current research interests include computational intelligence, group decision making, computing with words, information fusion, and government audit. He has authored the following book published by Springer: Theory and Approaches of Group Decision Making with Uncertain Linguistic Expressions. He has contributed more than 30 research papers to professional journals.

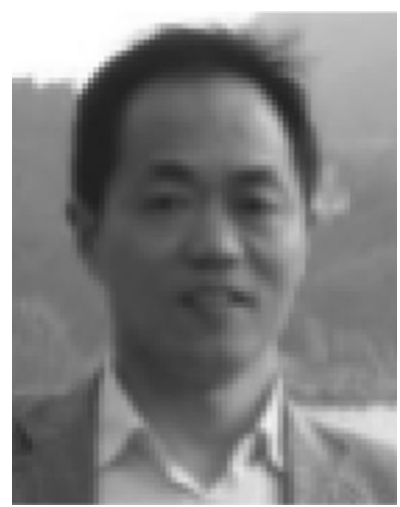

Zeshui Xu (M'08-SM'09F'18) received the Ph.D. degree in management science and engineering from Southeast University, Nanjing, China, in 2003. From April 2003 to May 2005, he was a Post-doctoral Researcher with the School of Economics and Management, Southeast University. From October 2005 to December 2007, he was a Postdoctoral Researcher with the School of Economics and Management, Tsinghua University, Beijing, China. He is currently a Professor with the Business School, Sichuan
University, Chengdu, China. He has authored the following books: Uncertain Multi-Attribute Decision Making: Methods and Applications (Springer, 2015), Intuitionistic Fuzzy Information Aggregation: Theory and Applications (Science Press and Springer, 2012), Linguistic Decision Making: Theory and Methods (Science Press and Springer, 2012), Intuitionistic Fuzzy Preference Modeling and Interactive Decision Making (Springer, 2013), Intuitionistic Fuzzy Aggregation and Clustering (Springer, 2013), and Hesitant Fuzzy Sets Theory (Springer, 2014). He has contributed more than 400 journal articles to professional journals. His current research interests include information fusion, group decision making, computing with words, and aggregation operators. Dr. Xu is an IFSA Fellow, IET Fellow, BCS Fellow, RSA Fellow, Distinguished Young Scholar of the National Natural Science Foundation of China, and the Chang Jiang Scholars of the Ministry of Education of China. His h-index is 86 . He has been selected as a 2014 Thomson Reuters Highly Cited Researcher (in the fields of Computer Science and Engineering, respectively) and also included in The World's Most Influential Scientific Minds 2014. He is the Advisory Member of the journal Granular Computing, the Associate Editor of Fuzzy Optimization and Decision Making, Journal of Intelligence Systems, the Section Editor of Asian Journal of Social and Economic Sciences, and the Chief Editor of Scholars Journal of Economics, Business and Management.He is also a member of the Editorial Boards of Information Fusion, Information: An International Journal, International Journal of Applied Management Science, International Journal of Data Analysis Techniques and Strategies, Journal of Applied and Computational Mathematics, International Journal of Research in Industrial Engineering, System Engineering Theory and Practice, Fuzzy Systems and Mathematics, Journal of Systems Engineering,and Chinese Journal of Management Science, etc. 